\title{
VAV2, a guanine nucleotide exchange factor for Rac1, regulates glucose-stimulated insulin secretion in pancreatic beta cells
}

\author{
Rajakrishnan Veluthakal ${ }^{1,3} \cdot$ Ragadeepthi Tunduguru $^{4}$. \\ Daleep Kumar Arora $^{5}$ - Vaibhav Sidarala ${ }^{1,2} \cdot$ Khadija Syeda $^{1,2}$ - Cornelis P. Vlaar ${ }^{6}$. \\ Debbie C. Thurmond $^{3,4}$ • Anjaneyulu Kowluru ${ }^{1,2,7}$
}

Received: 13 March 2015 / Accepted: 9 July 2015 / Published online: 31 July 2015

(C) Springer-Verlag (outside the USA) 2015

\begin{abstract}
Aims/hypothesis Rho GTPases (Ras-related C3 botulinum toxin substrate 1 [Rac1] and cell division cycle 42 [Cdc42]) have been shown to regulate glucose-stimulated insulin secretion (GSIS) via cytoskeletal remodelling, trafficking and fusion of insulin-secretory granules with the plasma membrane. GTP loading of these G proteins, which is facilitated by GDP/GTP exchange factors, is a requisite step in the regulation of downstream effector proteins. Guanine nucleotide exchange factor VAV2 (VAV2), a member of the Dbl family of proteins, has been identified as one of the GDP/GTP exchange factors for Rac1. Despite recent evidence on the regulatory
\end{abstract}

Electronic supplementary material The online version of this article (doi:10.1007/s00125-015-3707-4) contains peer-reviewed but unedited supplementary material, which is available to authorised users.

Anjaneyulu Kowluru akowluru@med.wayne.edu

1 Beta Cell Biochemistry Laboratory, John D. Dingell VA Medical Center, 4646 John R, Detroit, MI 48201, USA

2 Department of Pharmaceutical Sciences, Eugene Applebaum College of Pharmacy, Wayne State University, Detroit, MI, USA

3 Department of Pediatrics, Herman B Wells Center for Pediatric Research, Indiana University School of Medicine, Indianapolis, IN, USA

4 Department of Biochemistry and Molecular Biology, Indiana University School of Medicine, Indianapolis, IN, USA

5 Immunobiology and Cancer Research Program, Oklahoma Medical Research Foundation, Oklahoma City, OK, USA

6 Department of Pharmaceutical Sciences, School of Pharmacy, University of Puerto Rico, Medical Sciences Campus, San Juan, Puerto Rico

7 B-4237 Research Service, John D. Dingell VA Medical Center, 4646 John R, Detroit, MI 48201, USA roles of VAV2 in different cell types, roles of this guanine nucleotide exchange factor in the signalling events leading to GSIS remain undefined. Using immunological, short interfering RNA (siRNA), pharmacological and microscopic approaches we investigated the role of VAV2 in GSIS from islet beta cells.

Methods Co-localisation of Rac1 and VAV2 was determined by Triton X-114 phase partition and confocal microscopy. Glucose-induced actin remodelling was quantified by live cell imaging using the LifeAct-GFP fluorescent biosensor. Rac1 activation was determined by $\mathrm{G}$ protein linked immunosorbent assay (G-LISA).

Results Western blotting indicated that VAV2 is expressed in INS-1 832/13 beta cells, normal rat islets and human islets. Vav2 siRNA markedly attenuated GSIS in INS-1 832/13 cells. Ehop-016, a newly discovered small molecule inhibitor of the VAV2-Rac1 interaction, or siRNA-mediated knockdown of VAV2 markedly attenuated glucose-induced Rac1 activation and GSIS in INS-1 832/13 cells. Pharmacological findings were recapitulated in primary rat islets. A high glucose concentration promoted co-localisation of Rac1 and VAV2. Realtime imaging in live cells indicated a significant inhibition of glucose-induced cortical actin remodelling by Ehop-016. Conclusions/Interpretation Our data provide the first evidence to implicate VAV2 in glucose-induced Rac1 activation, actin remodelling and GSIS in pancreatic beta cells.

Keywords Cytoskeleton - Insulin secretion - Pancreatic islet . Rac1 - VAV2
Abbreviations
Cdc42 Cell division cycle 42
GDI GDP-dissociation inhibitor
GEF Guanine nucleotide exchange factor 


$\begin{array}{ll}\text { GFP } & \text { Green fluorescent protein } \\ \text { GSIS } & \text { Glucose-stimulated insulin secretion } \\ \text { G-LISA } & \text { G protein linked immunosorbent assay } \\ \text { HG } & \text { High glucose } \\ \text { LG } & \text { Low glucose } \\ \text { LS } & \text { Low serum } \\ \text { Rac1 } & \text { Ras-related C3 botulinum toxin substrate 1 } \\ \text { SFK } & \text { Src family of tyrosine kinases } \\ \text { siRNA } & \text { Short interfering RNA } \\ \text { Tiam1 } & \text { T-lymphoma invasion and metastasis-inducing } \\ & \text { protein 1 } \\ \text { VAV2 } & \text { Guanine nucleotide exchange factor VAV2 }\end{array}$

\section{Introduction}

Insulin secretion from pancreatic beta cells is principally regulated by ambient glucose concentrations. However, potential cellular mechanisms underlying the stimulus-secretion coupling of glucose-stimulated insulin secretion (GSIS) are only partially understood. GSIS occurs largely via the generation of soluble second messengers, such as cyclic nucleotides and biologically active lipids, as well as an increase in intracellular calcium concentrations [1-4].

Small $\mathrm{G}$ proteins (Ras-related $\mathrm{C} 3$ botulinum toxin substrate 1 [Rac1] and cell division cycle 42 [Cdc42]) play key regulatory roles in cytoskeletal remodelling to promote mobilisation of secretory granules to the plasma membrane for fusion and the release of their cargo into circulation [5-7]. Published evidence affirms the involvement of $\mathrm{Cdc} 42$, Rac1 and Arf6 in GSIS [5-11]. Functional activation-deactivation of these G proteins is modulated by a variety of regulatory factors/proteins. At least three classes of such factors/proteins have been described, including: (1) guanine nucleotide exchange factors (GEFs), which facilitate the conversion of GDP-bound (inactive) forms of G proteins to their GTP-bound (active) forms; (2) GDP-dissociation inhibitors (GDIs), which prevent the dissociation of GDP from G proteins, and hence are considered inhibitory in the $\mathrm{G}$ protein activation cascade; and (3) GTPase activating proteins, which promote the conversion of the GTP-bound, functionally active $\mathrm{G}$ proteins to their respective GDP-bound inactive conformation by activating the intrinsic GTPase activity of candidate G proteins to complete the activation-deactivation cycle [6]. Several recent studies have identified and studied GEFs for Cdc42, Rac1 and Arf6, which are implicated in GSIS. They include T-lymphoma invasion and metastasis-inducing protein 1 (Tiam1), Rho guanine nucleotide exchange factor 7 (Cool-1/ßPIX) and cytohesin-2 (ARNO), which have been shown to increase GTP loading onto Rac1, Cdc42 and Arf6, respectively [6, $10,12,13]$. Furthermore, Rho-GDI has been shown to regulate $\mathrm{Cdc} 42$ and Rac1 signalling pathways in islet beta cells, leading to GSIS $[14,15]$.
In addition to Tiam1 (a GEF for Rac1), guanine nucleotide exchange factor VAV2 (VAV2) has been identified as a GEF in the regulation of Rac1 functions in many cell types [16-18]. VAV2 belongs to the mammalian Vav family of GEFs: Vav1 is exclusively expressed only in haematopoietic cells [19], whereas VAV2 and VAV3 are ubiquitously expressed [19]. VAV2 is relatively unstudied. In general, there is little information on its role in cellular function; in particular, its expression levels and role in regulating GSIS have not been examined in islet beta cells. Several domains that span the VAV2 protein are important for various signalling events and also exhibit tyrosine-phosphorylation-dependent GEF activity [20-22]. Several Src family tyrosine kinases (SFKs) such as Lck and Fyn [23, 24], Syk family tyrosine kinases (Syk and Zap70) [24, 25], and receptor tyrosine kinases [26, 27] have been implicated as mediators of VAV2 tyrosine phosphorylation. Recent studies have shown that activation of tyrosineprotein kinase Yes (an Src family kinase) is indispensable for Cdc42 activation in a glucose-specific manner in pancreatic beta cells [28]. The aim of the current study, therefore, was to understand the roles of VAV2 in islet function, including GSIS. We addressed this by quantifying GSIS in beta cells in which VAV2 expression was suppressed via a short interfering RNA (siRNA) approach. Findings from these experiments were further validated by a pharmacological approach involving Ehop-016, a novel small molecule inhibitor of VAV2-Rac1 interaction (electronic supplementary material [ESM] Fig. 1) [29]. Collectively, findings from these investigations implicate VAV2, and perhaps the glucose-induced tyrosine phosphorylation of VAV2, in the process of Rac1mediated cortical actin remodelling and GSIS.

\section{Methods}

Antisera against VAV2 and $\beta$-actin were obtained from Santa Cruz Biotechnology (Santa Cruz, CA, USA) and SigmaAldrich (St Louis, MO, USA), respectively. Enhanced chemiluminescence kits were obtained from Amersham Biosciences (Piscataway, NJ, USA). Rac1 antiserum was obtained from BD Transduction Laboratories (San Jose, CA, USA). Donkey anti-mouse Alexa Fluor 488 and donkey anti-rabbit Alexa Fluor 568 conjugated secondary antibodies were obtained from Life technologies (Grand Island, NY, USA). The rat insulin ELISA kit was obtained from American Laboratory Products Co (Windham, NH, USA). The G protein linked immunosorbent assay (G-LISA) kit used for the Rac1 activation assay was obtained from Cytoskeleton (Denver, CO, USA). Vav2 siRNA and scrambled siRNA were obtained from Thermo Scientific (Waltham, MA, USA). Ehop-016 was synthesised as previously described [29]. SU6656 was obtained from Calbiochem (San Diego, CA, USA). 
INS-1 832/13 cells, rat islets and human islets INS-1 832/13 cells were cultured as previously described [8, 10]. Islets from normal male Sprague Dawley rats $(\sim 6$ weeks old; Harlan Laboratories, Oxford, MI, USA) were isolated by the collagenase digestion method [8, 10]. All protocols were reviewed and approved by the Institutional Animal Care and Use Committee at Wayne State University. Human islets were obtained from PRODO Laboratories (Irvine, CA, USA). Studies involving human islets were conducted according to the guidelines established by the US Department of Health and Human Services/NIH and approved by the Biosafety Committee at the John D. Dingell VA Medical Center.

Insulin release assay INS-1 $832 / 13$ cells or rat islets were incubated overnight with either vehicle or Ehop-016 ( $5 \mu \mathrm{mol} / \mathrm{l}$ ) in low glucose (LG; $2.5 \mathrm{mmol} / \mathrm{l}$ ) and low serum (LS; 2.5\%) medium. Following a $60 \mathrm{~min}$ pre-incubation in $\mathrm{KRB}$, the cells were further stimulated with either LG (2.5 mmol/1) or high glucose (HG; $20 \mathrm{mmol} / \mathrm{l})$ for $45 \mathrm{~min}$ at $37^{\circ} \mathrm{C}$ with or without Ehop-016. Insulin released was quantified by ELISA $[8,10]$.

Transfection studies with siRNA INS-1 832/13 cells were transfected with ON-TARGETplus SMARTpool Vav2 siRNA or scrambled siRNA at a final concentration of $80 \mathrm{nmol} / 1$ using Lipofectamine RNAiMAX transfection reagent (Life technologies, Grand Island, NY, USA). The efficiency of VAV2 knockdown was determined by western blot analysis at $48 \mathrm{~h}$ post-transfection.

Rac1 activation assay Activated Rac1 was quantified by the Rac1 activation G-LISA assay kit in INS-1 832/13 cells treated with Ehop-016 or transfected with Vav2 siRNA [30].

Live cell imaging studies INS-1 832/13 cells were seeded on MatTek (Ashland, MA, USA) glass bottom culture dishes at a density of 400,000 cells per $35 \mathrm{~mm}$ dish. At $~ 50 \%$ confluency, cells were transfected with the LifeAct-GFP plasmid using Lipofectamine 2000 transfection reagent (Invitrogen, Carlsbad, CA, USA) [31]. Live cell imaging was performed on cells at $48 \mathrm{~h}$ post-transfection. Briefly, cells were preincubated overnight in LS-LG medium with or without Ehop-016 (5 $\mu \mathrm{mol} / \mathrm{l})$. After $24 \mathrm{~h}$, cells were pre-incubated in KRB buffer for $1 \mathrm{~h}$ with or without Ehop-016. Images were captured every $2 \mathrm{~min}$, starting from 0-20 min after the addition of $20 \mathrm{mmol} / 1$ glucose [31].

Subcellular fractionation: Triton $\mathrm{X}-114$ phase partitioning assay Lysates derived from INS-1 832/13 cells treated with LG or HG were centrifuged at $100,000 \mathrm{~g}$ for $60 \mathrm{~min}$ at $4^{\circ} \mathrm{C}$ to obtain total membrane (pellet) and soluble (supernatant) fractions. The hydrophilic and hydrophobic phases of the total membrane fractions were isolated using Triton X-114 as previously described [14].

VAV2-Rac1 co-localisation by confocal immunofluorescence microscopy INS-1 $832 / 13$ cells were plated on glass coverslips. After $24 \mathrm{~h}$, the cells were incubated in LS-LG medium overnight and then with KRB for $1 \mathrm{~h}$ prior to incubation with $\mathrm{LG}$ or $\mathrm{HG}$ for $15 \mathrm{~min}$ at $37^{\circ} \mathrm{C}$. Following this, cells were washed in PBS, fixed in ice-cold methanol for $20 \mathrm{~min}$ at $-20^{\circ} \mathrm{C}$ and then washed in PBS. Non-specific binding sites were first blocked for $1 \mathrm{~h}$ with $5 \%$ donkey serum and then cells were incubated with mouse anti-Rac1 monoclonal antibody (1:200 dilution) for $1 \mathrm{~h}$, washed, and then incubated overnight with rabbit anti-VAV2 polyclonal antibody (1:100 dilution). After washing in PBS, cells were incubated for $1 \mathrm{~h}$ with Alexa Fluor 488 to detect Rac1. To detect VAV2, cells were incubated with Alexa Fluor 568. PBS with 5\% donkey serum was used for antibody dilution. Cells were then washed and proteins were visualised by confocal scanning laser microscopy, as previously described [9].

Statistical analysis The statistical significance of differences between control and experimental conditions was determined by the Student's $t$ test and ANOVA. A $p$ value of $<0.05$ was considered statistically significant.

\section{Results}

VAV2 is expressed in INS-1 832/13 cells, rodent and human islets and regulates GSIS Data shown in Fig. 1a suggest that VAV2 is expressed in INS-1 832/13 cells, rat islets and human islets. Levels of VAV2 in all three cells were comparable, as determined by protein to $\beta$-actin ratios. We next asked whether VAV2 regulates GSIS by quantifying the latter in INS-1 832/13 cells in which endogenous VAV2 expression was depleted via Vav2 siRNA. Data in Fig. 1b, c demonstrate a $\sim 60 \%$ reduction in VAV2 in Vav2 siRNA transfected cells relative to cells transfected with scrambled siRNA. More importantly, GSIS is markedly reduced in these cells following knockdown of VAV2 (Fig. 1d), suggesting that VAV2 plays a regulatory role in GSIS.

Ehop-016 attenuates GSIS from INS-1 832/13 cells and normal rodent islets We further examined the roles of VAV2 in GSIS in INS-1 832/13 cells and normal rat islets using Ehop-016, a novel small molecule inhibitor of the VAV2-Rac1 GTPase interaction (ESM Fig. 1) [29]. We quantified GSIS in INS-1 832/13 cells (Fig. 2a) and normal rat islets (Fig. 2b) incubated with or without Ehop-016. Our findings indicated significant inhibition of GSIS by Ehop-016 in both cell types studied, further confirming our observations described in Fig. 1. Together, the data in Figs 1 and 2 suggest 
a
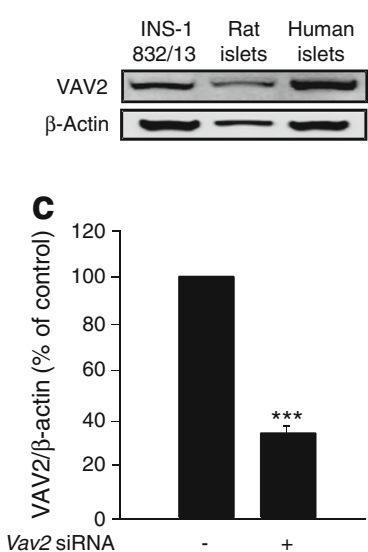

b

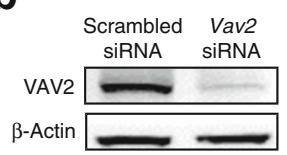

d

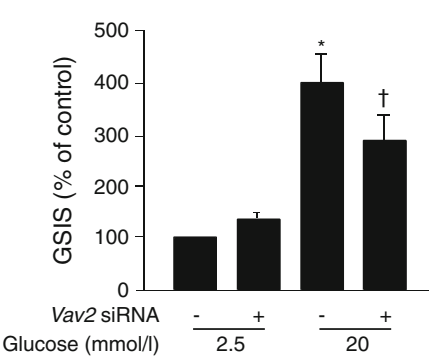

Fig. 1 SiRNA-mediated knockdown of VAV2 attenuates GSIS in INS-1 832/13 cells. (a) VAV2 levels in INS-1 832/13 cells and rat and human islets. (b) Cells were transfected with either scrambled or Vav2 siRNA for $48 \mathrm{~h}$. Western blotting indicated efficient knockdown of VAV2. (c) Densitometric analysis showed a $\sim 60 \%$ reduction in VAV2 levels in cells transfected with Vav2 siRNA. Data represent means \pm SEM $(n=3)$ and are expressed as percentage of control. ${ }^{* * *} p<0.001$ vs scrambled siRNA. (d) Following transfection, INS-1 832/13 cells were stimulated with HG and insulin released was quantified by ELISA. Data represent means $\pm \operatorname{SEM}(n=3)$ and are expressed as percentage of control. ${ }^{*} p<0.05$ vs LG with scrambled siRNA, ${ }^{\dagger} p<0.05$ vs HG with scrambled siRNA

that VAV2 plays a regulatory role in GSIS. These data further indicate that INS-1 832/13 cells reliably model VAV2mediated regulation of GSIS as exhibited by primary rat islets.

Inhibition of VAV2 suppresses glucose-induced Rac1 activation in INS-1 832/13 cells A large number of studies have demonstrated that GSIS involves activation of Arf6, Cdc42 and Rac1, with these activation events being necessary steps for the cytoskeletal remodelling to facilitate the movement of granules from the intracellular location towards the plasma membrane [5-7]. Since VAV2 is a known regulator of Rac1 [18-20], we next asked whether glucose-induced activation of Rac1 is sensitive to inhibition of VAV2. To address this, we quantified glucose-induced Rac1 activation in INS-1 832/13 cells incubated in the absence or presence of Ehop-016. Data in Fig. 2c indicate a significant increase in Rac1 activation by stimulatory concentrations of glucose. Furthermore, Ehop016 completely inhibited glucose-induced activation of Rac1 under these conditions, without significantly affecting basal levels of Rac1 activation (Fig. 2c). Furthermore, we noticed significant inhibition of glucose-induced Rac1 activation following siRNA-mediated knockdown of VAV2 (Fig. 2d). Collectively, our findings in INS-1 832/13 cells and normal rodent islets suggest that GSIS involves an activation step involving VAV2 that is upstream of Rac1 activation.

We also assessed the regulatory roles of VAV2 in $\mathrm{KCl}-$ induced insulin secretion in INS-1 832/13 cells, and observed that $\mathrm{KCl}$-induced insulin secretion is modestly inhibited by

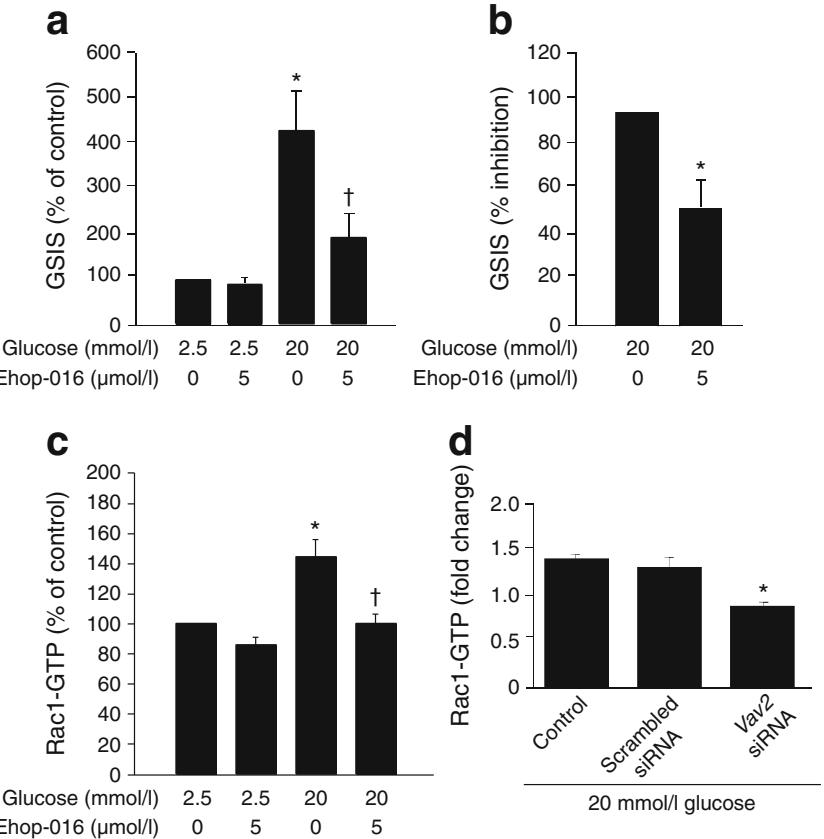

Fig. 2 VAV2 mediates glucose-induced insulin secretion and Rac1 activation. (a) INS-1 832/13 cells were incubated in LS-LG medium with or without Ehop-016 (5 $\mu \mathrm{mol} / \mathrm{l})$ or vehicle and stimulated further with $\mathrm{HG}$. Insulin released was quantified by ELISA. Data represent means \pm SEM $(n=3)$ and are expressed as percentage of control. ${ }^{*} p<0.05$ vs $2.5 \mathrm{mmol} / 1$ glucose without Ehop-016, ${ }^{\dagger} p<0.05$ vs HG without Ehop-016. (b) Normal rat islets were incubated overnight with or without Ehop-016 $(5 \mu \mathrm{mol} / 1)$ and stimulated with HG. Insulin released was quantified by ELISA. Data represent means $\pm \operatorname{SEM}(n=3)$ and are expressed as percentage inhibition of GSIS by Ehop-016. ${ }^{*} p<0.05$ vs HG without Ehop-016. (c) INS-1 832/13 cells were incubated overnight in LS-LG medium with or without Ehop-016 (5 $\mu \mathrm{mol} / \mathrm{l})$ and stimulated (15 min) with HG. Activated Rac1 was quantified by G-LISA. Data represent means \pm SEM ( $n=$ 3 ) and are expressed as percentage of control. $* p<0.05$ vs LG without Ehop-016, ${ }^{\dagger} p<0.05$ vs HG without Ehop-016. (d) INS-1 832/13 cells were transfected with either scrambled siRNA or Vav2 siRNA for $48 \mathrm{~h}$ and stimulated with either $\mathrm{LG}$ or $\mathrm{HG}$ for $15 \mathrm{~min}$ in $\mathrm{KRB}$ at $37^{\circ} \mathrm{C}$. Activated Rac1 was quantified by G-LISA. Data represent means \pm SEM from three independent experiments and are expressed as the fold change. $* p<0.05$ vs HG with or without scrambled siRNA

Ehop-016 (ESM Fig. 2). More importantly, unlike stimulatory glucose (Fig. 2d), $\mathrm{KCl}$ failed to activate Rac1 (ESM Fig. 2) in both control and Vav2 siRNA transfected cells (ESM Fig. 2). Even though Rac1 activity was lower than under basal conditions (i.e. control cells treated with LG) in cells transfected with scrambled and Vav2 siRNA, this was not due to decreased levels of Rac1 in these cells (additional data not shown). These observations are compatible with previous observations that Rac1 activation is dispensable for $\mathrm{KCl}$-induced insulin secretion $[6,8]$.

Glucose promotes the association between VAV2 and Rac1: evidence from Triton $\mathrm{X}-114$ phase partition assay and immunofluorescence methods GSIS involves the mobilisation of Rac1 and $\mathrm{Cdc} 42$ to the membrane for interaction with various effector proteins. Therefore, we investigated 
the subcellular association (e.g. targeting) of Rac1 and VAV2 with hydrophilic and hydrophobic compartments in INS-1 $832 / 13$ cells treated with or without stimulatory glucose. Triton X-114 phase partitioning was used to isolate the cytosolic and membrane compartments, and to further segregate the hydrophobic and hydrophilic compartments comprising the total membrane fraction (ESM Fig. 3), as previously described and validated [14]. Data in Fig. 3a suggest that VAV2 is distributed in both the hydrophobic and hydrophilic compartments under basal as well as high-glucose exposure conditions. In contrast, Rac1 was predominantly associated with the cytosolic compartment under basal glucose conditions, and glucose stimulation promoted the association of Rac 1 with the hydrophilic phase of the membrane (Fig. 3b). It is well established that GDI binds Rho GTPases (e.g. Rac1) through their C-terminal lipid anchors [32] and retains them in the cytosol $[32,33]$. Available evidence also suggests that activation of Rho GTPases occurs in the cytosol, leading to their membrane association following dissociation from the cytosolic GDI protein $[14,15]$. Our findings suggest that VAV2-mediated activation of Rac1 might occur in the cytosolic compartment followed by its translocation to the hydrophilic phase of the membrane in glucose-stimulated beta cells. These findings were further confirmed by confocal immunofluorescence microscopy (Fig. 3c, d). Compatible with the translocation data shown in Fig. 3a, b, we noted increased co-localisation of VAV2 (in red) with Rac1 (in green) in the cytosolic compartment (indicated by yellow colour; arrows). Thus, our findings from two distinct methods support the hypothesis that stimulatory concentrations of glucose promote co-localisation of VAV2 and Rac1.

The VAV2-Rac1 axis mediates glucose-induced filamentous actin (F-actin) remodelling in beta cells Previous studies have demonstrated that the second-phase of insulin secretion, requires the movement of granules from intracellular site to the plasma membrane, which involves reorganisation of the F-actin cytoskeletal network barrier; Rac1 is implicated in second-phase GSIS [15]. To determine the requirement of

Fig. 3 Glucose promotes an association between VAV2 and Rac1 in INS-1 832/13 cells. (a, b) Cells were incubated overnight in LS-LG medium and then stimulated (15 min) further with HG. The total soluble fraction and hydrophilic (HPL) and hydrophobic (HPB) phases of the particulate fractions were isolated using the Triton X-114 phase partitioning method. The abundance of VAV2 (a) and Rac1 (b) in these fractions was determined by western blotting. Data represent means \pm SEM $(n=3)$ and are expressed as the fold change over LG. ${ }^{*} p<0.05$ vs LG. NS, not significant. (c, d) Cells were incubated overnight in LG-LS medium and then stimulated further with $\mathrm{HG}$ for $15 \mathrm{~min}$ in $\mathrm{KRB}$ at $37^{\circ} \mathrm{C}$. The expression patterns of both Rac1 (green) and VAV2 (red) are similar under both LG (c) and HG (d) conditions, whereas regions of yellow colour appearing as a distinct punctate pattern (indicated by arrows) in HG-treated cells demonstrate the possibility of Rac1 and VAV2 colocalisation in the cytosolic compartment. Scale bars, $13 \mu \mathrm{m}$ a

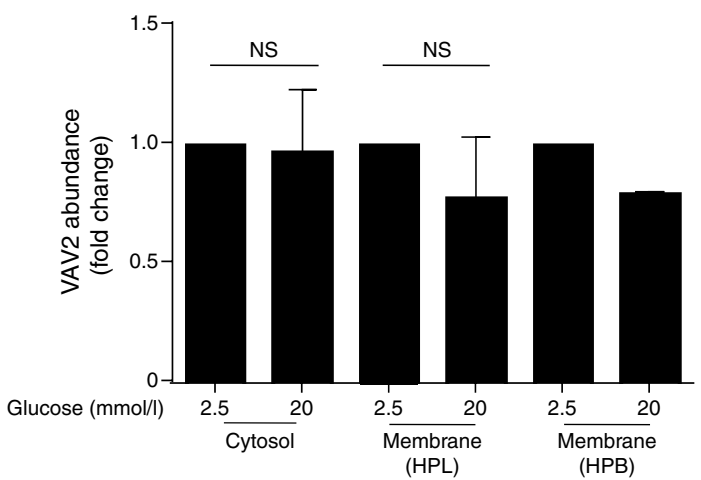

b

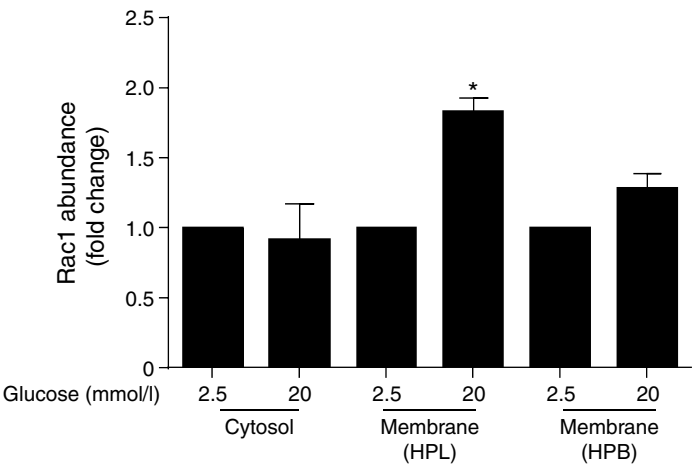

C

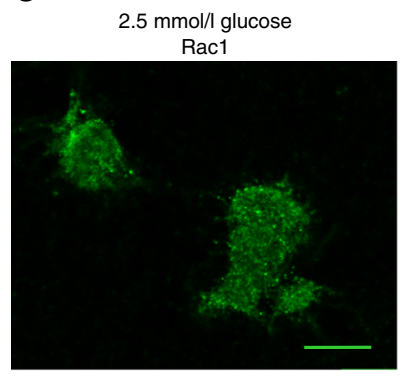

VAV2

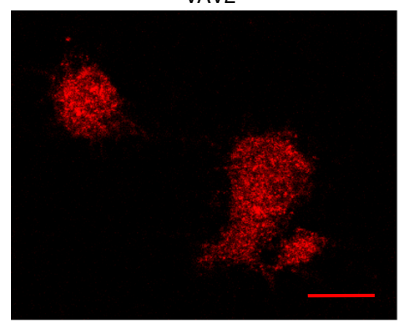

Merge

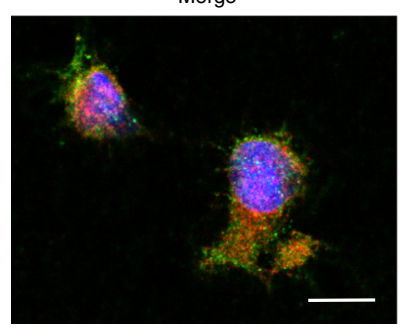

d

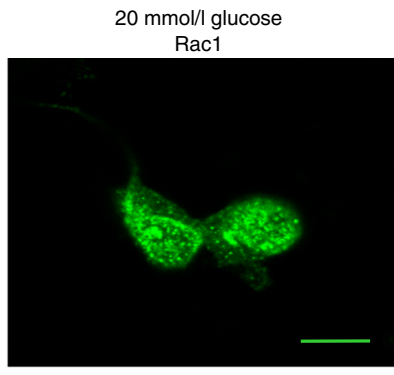

VAV2

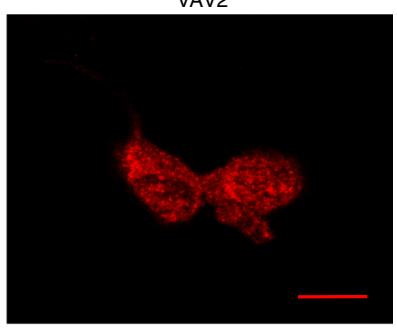

Merge

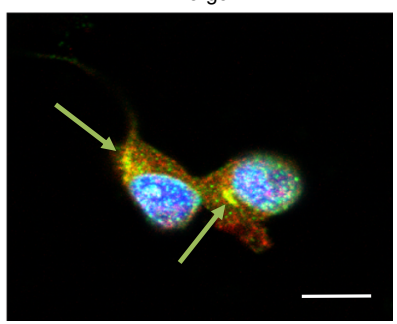


VAV2 in the reorganisation of cortical F-actin, the LifeActGFP biosensor of F-actin was used as an imaging agent in INS-1 832/13 cells treated with Ehop-016 during GSIS. LifeAct-GFP is a 17 amino acid peptide from the Abp140 actin-binding protein linked to the N-terminus of green fluorescent protein (GFP) previously shown to specifically bind to F-actin without significantly affecting F-actin dynamics $[34$, $35]$. In INS- $1832 / 13$ beta cells, the glucose-induced depolymerisation of F-actin (i.e. disappearance of F-actin staining) became evident within $5 \mathrm{~min}$ and continued progressively for up to $\sim 20 \mathrm{~min}$ (Fig. 4a; ESM Videos 1, 2). In contrast, Ehop-016 abolished the F-actin depolymerisation, as observed by intact F-actin staining in the entire cell (Fig. 4b; ESM Videos 3, 4). These data support a role for VAV2-induced Rac1 activation in actin depolymerisation in beta cells, consistent with its role in facilitating insulin secretion.

SU6656, a tyrosine kinase inhibitor, attenuates glucoseinduced phosphorylation of VAV2 in beta cells Several previous studies have demonstrated that tyrosine phosphorylation of VAV2 is important for GDP/GTP exchange activity on downstream targets such as Rac1 [21]. To test this hypothesis in beta cells, we utilised SU6656, a selective inhibitor of SFKs. We have observed a significant increase in phosphorylation of VAV2 under stimulatory concentrations of glucose. Treatment with SU6656 abolished glucose-induced VAV2 phosphorylation (Fig. 5), suggesting that upstream SFK activation by glucose is important for the VAV2-mediated Rac1 activation signalling step. Taken together, our data suggest that VAV2-mediated activation of Rac1 represents one of the key signalling steps involved in actin cytoskeleton remodelling and facilitation of GSIS from pancreatic beta cell.

\section{Discussion}

The main objective of this study was to investigate the role of VAV2, a known GEF for Rac1, in GSIS. Our findings suggest that the molecular biological and pharmacological inhibition of VAV2 results in the inhibition of glucose-induced activation of Rac1 and GSIS in INS-1 832/13 cells and primary rodent islets, thus suggesting novel roles for VAV2-Rac1 signalling in GSIS. It is well established that $\mathrm{Cdc} 42$ and Rac1 play essential roles in cytoskeletal remodelling, vesicular transport and fusion with the plasma membrane [5, 6]. Using a variety of experimental approaches, we previously described regulatory roles for Rac1 in islet function and GSIS [6, 8, 36-38]. We demonstrated that inhibition of Rac1 function significantly attenuated glucose-, but not KCl-induced insulin secretion in clonal beta cells and rodent islets $[8,38]$. We also reported that pharmacological or molecular biological inhibition of Tiam1, a known GEF for Rac1, significantly inhibits GSIS [12]. These data suggested that Tiam1 is one of the GEFs involved in glucose-induced activation of Rac1 and insulin secretion.

What then are the roles of VAV2 in this signalling cascade? Our current findings clearly raise an interesting possibility that other GEFs, such as VAV2, regulate GSIS. It is likely that both Tiam1 and VAV2 exert distinct regulatory functions leading to the activation of Rac1 and downstream signalling steps,

a
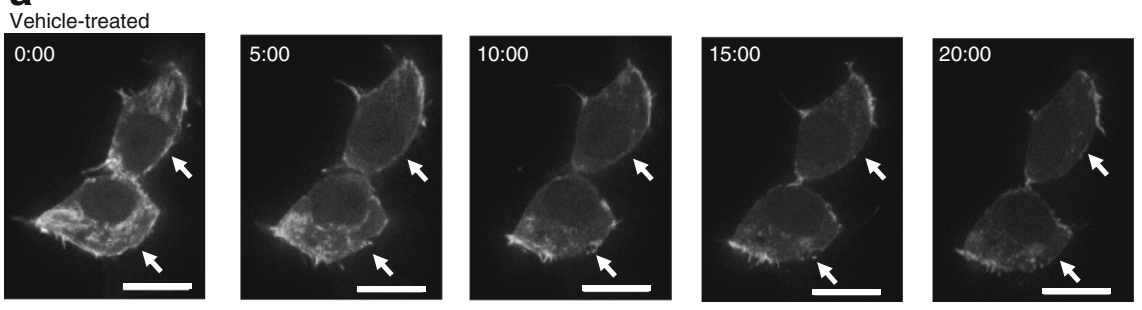

b
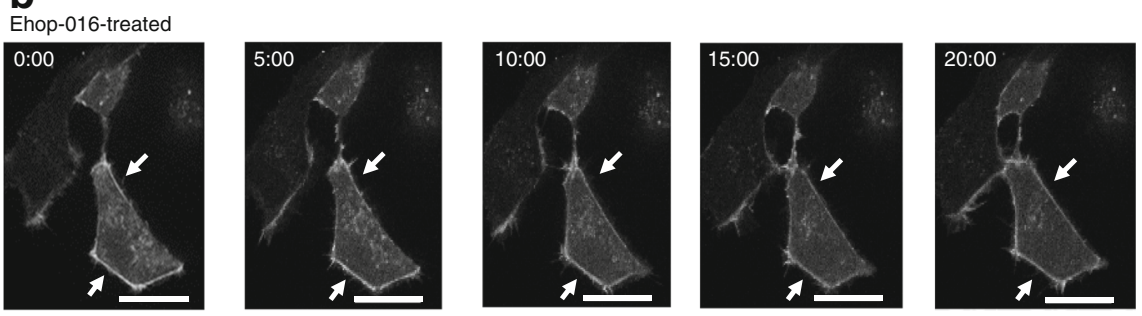

Fig. 4 Ehop-016 inhibits glucose-induced actin remodelling in INS-1 $832 / 13$ cells. $(\mathbf{a}, \mathbf{b})$ Cells were transfected with LifeAct-GFP and $48 \mathrm{~h}$ later were pre-incubated overnight in LG-LS RPMI 1640 medium. The next day, cells were pre-treated with DMSO (a) or Ehop-016 (b) in KRB for $1 \mathrm{~h}$ and live cell imaging was captured every $2 \mathrm{~min}$ for $20 \mathrm{~min}$ starting directly after the addition of $\mathrm{HG}$. Representative images from three experiments consisting of at least 12 cells for each condition are shown. A total of 24 cells were analysed for each condition. In (a) arrows demonstrate the disappearance of cortical actin when cells were treated with $20 \mathrm{mmol} / \mathrm{l}$ glucose at different time intervals. In (b) arrows demonstrate intact cortical actin in cells treated with Ehop-016 and then exposed to $20 \mathrm{mmol} / \mathrm{l}$ glucose. Scale bars, $10 \mu \mathrm{m}$ 


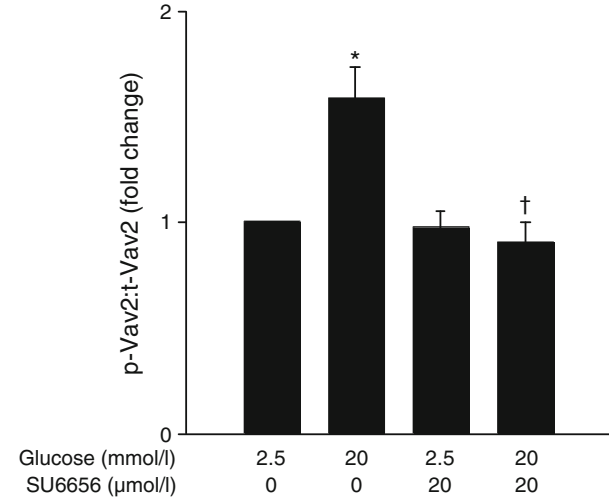

Fig. 5 Glucose induces tyrosine phosphorylation of VAV2 in INS-1 832/ 13 cells. Cells were incubated in LS-LG medium overnight prior to $1 \mathrm{~h}$ incubation with vehicle or SU6656 $(20 \mu \mathrm{mol} / \mathrm{l})$ in KRB. Cells were then stimulated with either $\mathrm{LG}$ or $\mathrm{HG}$ for $10 \mathrm{~min}$ in $\mathrm{KRB}$ at $37^{\circ} \mathrm{C}$ in the presence or absence of SU6656. Phosphorylation of VAV2 was detected by western blotting, using anti-phospho-VAV2 serum and band intensities were quantified by densitometry. Data represent means $\pm \operatorname{SEM}(n=3)$ and are expressed as the fold change in the phospho-VAV2:total VAV2 ratio. ${ }^{*} p<0.05$ vs LG without SU6656, ${ }^{\dagger} p<0.05$ vs HG without SU6656

including activation of the phagocyte-like NADPH oxidase (Nox2) leading to GSIS [6, 38, 39]. Based on compelling experimental evidence, it was concluded that Nox 2 is one of the regulatory proteins involved in insulin secretion [6, 38-41]. Rac1 is one of the components of the Nox2 holoenzyme, and it has been shown that Rac1 associates with other members of this holoenzyme upon GTP binding and activation $[6,30,38]$. Therefore, we propose that both Tiam1 and VAV2 serve as GEFs for Rac1 in mediating GSIS. It is noteworthy that pharmacological inhibition of both the Tiam1Rac1 and VAV2-Rac1 pathways using NSC23766 and Ehop-016, respectively, yielded much greater inhibition of GSIS compared with inhibition of either of these pathways alone (ESM Fig. 4). These data indicate independent roles for these pathways (i.e. different downstream signalling events) in the induction of GSIS. Additional studies are needed to further validate this model. Liu and associates suggested novel regulation of Rac1 and redox signalling in response to fluid stress in endothelial cells [42]. They reported the concerted actions of Tiam1 and VAV2 in linking components of the polarity complex (vascular endothelial-cadherin-neutrophil cytosol factor 2 [p67phox]-partitioning defective 3 homolog [Par3]) to the NADPH oxidase module. Data from these investigations suggest that VAV2 is required for GTP loading onto Rac1, whereas Tiam1 serves as an adaptor protein for the polarity complex, which directs the localised activation of Rac1. Thus, it is likely that the Tiam1/VAV2 module regulates GSIS at different levels via regulating distinct signalling steps.

Emerging evidence suggests that the regulation of Tiam1 and VAV2 functions by tyrosine phosphorylation is catalysed by SFKs. For example, using pharmacological and molecular biological approaches, Servitja and associates demonstrated regulatory roles for Tiam1 and VAV2 in Src-induced cell transformation via regulation of Rac1 activation [43]. Earlier studies pointed out that phosphorylation of Vav on tyrosine residues [19-22] activates its GEF activity [26, 27]. The phosphorylation step is mediated by the Vav structural domain (Src homology 2), which facilitates the interaction of Vav proteins with membrane and cytoplasmic tyrosine kinases [19-22]. Our current studies demonstrate significant inhibition of glucose-induced VAV2 phosphorylation by SU6656. Indeed, recent investigations by Yoder et al [28] provide compelling evidence for YES tyrosine kinase activation as an early signalling event $(\sim 1 \mathrm{~min})$ involved in the activation of the small $\mathrm{G}$ protein $\mathrm{Cdc} 42(\sim 3 \mathrm{~min})$ in glucose-stimulated islet beta cell. Likewise, studies of Garrett et al have demonstrated a requirement for tyrosine phosphorylation of VAV2 in vascular endothelial growth factor-induced Rac1 activation in endothelial cells [44]. Together, these findings implicate a tyrosine phosphorylation step upstream to the activating GEFs (Tiam1/VAV2) for Rac1 activation and associated downstream signalling events. It is also likely that phosphorylation of VAV could be mediated via binding of the VAV2 pleckstrin homology domain region to biologically active lipid second messenger products of phosphatidylinositol-3-kinase [45] because these lipid second messengers are known to promote activation and translocation of Rac1 in beta cells [46]. Future studies are needed to test these putative mechanisms to more precisely determine the molecular roles for the Tiam1/ VAV2 axis in pancreatic beta cells.

Our current findings also suggest that VAV2-mediated Rac1 activation is important for actin cytoskeletal reorganisation and mobilisation of insulin granules towards the plasma membrane for their fusion and release. In the pancreatic beta cell, actin microfilaments juxtaposed to or beneath the plasma membrane are thought to restrict the localisation of secretory vesicles to release sites in the absence of stimulatory levels of glucose. Our data might suggest that VAV2-Rac1 binding is directly required for glucose-induced actin depolymerisation. Alternatively, these data may suggest that VAV2-Rac1 activation is downstream of events related to Cdc42 and PAK1 activation, both of which occur within the first $5 \mathrm{~min}$ of GSIS and are required for actin remodelling in the beta cell. Indeed, Cdc42 activation occurs downstream of YES kinase activation, an SFK-mediated tyrosine phosphorylation event. In conclusion, our findings provide the first evidence in real time for a VAV2-Rac1 signalling axis in actin cytoskeletal remodelling and GSIS in isolated beta cells, and form the basis for future investigations to precisely define the roles of this Tiam1/VAV2-Rac1 pathway in physiological insulin secretion in the islet.

Acknowledgements We thank L. Philipson (University of Chicago, Chicago, IL, USA) for providing the LifeAct-GFP and C. Newgard, (Duke University Medical Center, Durham, NC, USA) for providing INS-1 832/13 cells. 
Funding This research was supported in part by a Merit Review award to AK (1I01BX002801) from the Department of Veterans Affairs and awards from the National Institutes of Health to AK (DK94201 and EY022230), DCT (DK67912 and DK102233) and from the JDRF to AK (5-2012-257) and DCT (17-2013-454). AK is also the recipient of a Senior Research Career Scientist Award from the Department of Veterans Affairs (13S-RCS-006). RT is the recipient of an American Heart Association pre-doctoral fellowship.

Duality of interest The authors declare that there is no duality of interest associated with this manuscript.

Contribution statement AK, DCT, RV and CPV were responsible for the experimental plan, literature search, and manuscript writing and editing. DKA, RV, VS, RT and KS performed the experiments and analysed the data. RT, DKA, VS and KS also assisted AK in drafting the original manuscript and revising the article. All authors read and approved the final version of the manuscript. AK is responsible for the integrity of the work as a whole.

\section{References}

1. Jitrapakdee S, Wutthisathapornchai A, Wallace JC, MacDonald MJ (2010) Regulation of insulin secretion: role of mitochondrial signaling. Diabetologia 53:1019-1032

2. Prentki M, Matschinsky FM, Madiraju SR (2013) Metabolic signaling in fuel-induced insulin secretion. Cell Metab 18:162-185

3. Berggren PO, Leibiger IB (2006) Novel aspects on signal transduction in the pancreatic beta cell. Nutr Metab Cardiovasc Dis 16(suppl 1):S7-S10

4. Komatsu M, Takei M, Ishii H, Sato Y (2013) Glucose-stimulated insulin secretion: a newer perspective. J Diabetes Investig 4:511-516

5. Wang Z, Thurmond DC (2009) Mechanisms of biphasic insulin granule exocytosis-roles of cytoskeleton, small GTPases and SNARE proteins. J Cell Sci 122:893-903

6. Kowluru A (2010) Small G proteins in islet $\beta$-cell function. Endocr Rev 31:52-78

7. Kalwat MA, Thurmond DC (2013) Signaling mechanisms of glucose-induced F-actin remodeling in pancreatic islet $\beta$ cells. Exp Mol Med 45:1-12

8. Veluthakal R, Kaur H, Goalstone M, Kowluru A (2007) Dominantnegative alpha-subunit of farnesyl-and geranyltransferase inhibits glucose-stimulated, but not $\mathrm{KCl}$-stimulated, insulin secretion in INS 832/13 cells. Diabetes 56:204-210

9. Wang Z, Oh E, Thurmond DC (2007) Glucose-stimulated Cdc42 signaling is essential for the second phase of insulin secretion. J Biol Chem 282:9536-9546

10. Jayaram B, Syed I, Kyathanahalli CN, Rhodes CJ, Kowluru A (2011) Arf nucleotide binding site opener [ARNO] promotes sequential activation of Arf6, cdc42 and Rac1 and insulin secretion in INS 832/13 beta-cells and rat islets. Biochem Pharmacol 81:1016-1027

11. Lawrence JT, Birnbaum MJ (2003) ADP-ribosylation factor 6 regulates insulin secretion through plasma membrane phosphatidylinositol 4,5-biphosphate. Proc Natl Acad Sci U S A 100:13320-13325

12. Veluthakal R, Madathilperambil SV, McDonald P, Olson LK, Kowluru A (2009) Regulatory roles for Tiam1, a guanine nucleotide exchange factor for Rac1, in glucose-stimulated insulin secretion in pancreatic beta-cells. Biochem Pharmacol 77:101-113

13. Kepner EM, Yoder SM, Oh E et al (2011) Cool-1/ßPIX functions as a guanine nucleotide exchange factor in the cycling of $\mathrm{Cdc} 42$ to regulate insulin secretion. Am J Physiol Endocrinol Metab 301:1072-1080

14. Kowluru A, Veluthakal R (2005) Rho guanosine diphosphatedissociation inhibitor plays a negative modulatory role in glucosestimulated insulin secretion. Diabetes 54:3523-3529

15. Wang Z, Thurmond DC (2010) Differential phosphorylation of RhoGDI mediates the distinct cycling of Cdc42 and Rac1 to regulate second phase insulin secretion. J Biol Chem 285:6186-6197

16. Hornstein I, Alcover A, Katzav S (2004) Vav proteins, masters of the world of cytoskeleton organization. Cell Signal 16:1-11

17. Swat W, Fujikawa K (2005) The Vav family: at the crossroads of signaling. Immunol Res 32:259-265

18. Cook DR, Rossman KL, Der CJ (2013) Rho guanine nucleotide exchange factors: regulators of Rho GTPase activity in the development and disease. Oncogene 33:4021-4035

19. Bustelo XR (2000) Regulatory and signaling properties of the Vav family. Mol Cell Biol 20:1461-1477

20. Bustelo XR (1996) The VAV family of signal transduction molecules. Crit Rev Oncog 7:65-88

21. Crespo P, Schuebel KE, Ostrom AA, Gutkind JS, Bustelo XR (1997) Phosphotyrosine-dependent activation of Rac-1 GDP/GTP exchange by the vav proto-oncogene product. Nature 385:169-172

22. Schuebel KE, Movilla N, Rosa JL, Bustelo XR (1998) Phosphorylation-dependent and constitutive activation of Rho proteins by wild-type and oncogenic Vav-2. EMBO J 17:6608-6621

23. Han J, Das B, Wei W et al (1997) Lck regulates Vav activation of members of the Rho family of GTPases. Mol Cell Biol 17:1346-1353

24. Michel F, Grimaud L, Tuosto L, Acuto O (1998) Fyn and ZAP-70 are required for Vav phosphorylation in T cells stimulated by antigen-presenting cells. J Biol Chem 273:31932-31938

25. Deckert M, Tartare-Deckert S, Couture C, Mustelin T, Altman A (1996) Functional and physical interactions of Syk family kinases with the Vav proto-oncogene product. Immunity 5:591-604

26. Bustelo XR, Ledbetter JA, Barbacid M (1992) Product of vav proto-oncogene defines a new class of tyrosine protein kinase substrates. Nature 356:68-71

27. Margolis B, Hu P, Katzav S et al (1992) Tyrosine phosphorylation of vav proto-oncogene product containing $\mathrm{SH} 2$ domain and transcription factor motifs. Nature 356:71-74

28. Yoder SM, Dineen SL, Wang Z, Thurmond DC (2014) YES, a Src family kinase, is a proximal glucose-specific activator of cell division cycle control protein $42(\mathrm{Cdc} 42)$ in pancreatic islet $\beta$ cells. J Biol Chem 289:11476-11487

29. Montalvo-Ortiz BL, Castillo-Pichardo L, Hernandez E, HumphriesBickley T, De la Mota-Peynado A et al (2012) Characterization of Ehop-016, a novel small molecule inhibitor of Rac GTPase. J Biol Chem 287:13228-13238

30. Kowluru RA, Kowluru A, Veluthakal R et al (2014) Tiam1-Rac1 signaling axis-mediated activation of NADPH oxidase-2 initiates mitochondrial damage in the development of diabetic retinopathy. Diabetologia 57:1047-1056

31. Tunduguru R, Chiu TT, Ramalingam L, Elmendorf JS, Klip A, Thurmond DC (2014) Signaling of the p21-activated kinase (PAK1) coordinates insulin-stimulated actin remodeling and glucose uptake in skeletal muscle cell. Biochem Pharmacol 92:380-388

32. del Pozo MA, Kiosses WB, Alderson NB, Meller N, Hahn KM, Schwartz MA (2002) Integrins regulate GTP-Rac localized effector interactions through dissociation of Rho-GDI. Nat Cell Biol 4:232-239

33. Olofsson B (1999) Rho guanine dissociation inhibitors: pivotal molecules in cellular signalling. Cell Signal 11:545-554

34. Kalwat MA, Yoder SM, Wang Z, Thurmond DC (2013) A p21activated kinase (PAK1) signaling cascade coordinately regulates 
F-actin remodeling and insulin granule exocytosis in pancreatic $\beta$ cells. Biochem Pharmacol 85:808-816

35. Lopez JP, Turner JR, Philipson LH (2010) Glucose-induced ERM protein activation and translocation regulates insulin secretion. Am J Physiol Endocrinol Metab 299:E772-E785

36. Kowluru A, Li G, Rabaglia ME, Segu VB, Hofmann F et al (1997) Evidence for differential regulation of Rho subfamily of GTPbinding proteins in glucose-and calcium-induced insulin secretion from pancreatic beta-cells. Biochem Pharmacol 54:1097-1108

37. Kowluru A, Veluthakal R, Rhodes CJ, Kamath V, Syed I et al (2010) Protein farnesylation-dependent Raf/extracellular signalrelated kinase signaling links to cytoskeletal remodeling to facilitate glucose-induced insulin secretion in pancreatic beta-cells. Diabetes 59:967-977

38. Kowluru A (2011) Friendly, and not so friendly, roles of Rac1 in islet beta-cell function: lessons learnt from pharmacological and molecular biological approaches. Biochem Pharmacol 81:965-975

39. Syed I, Kyathanahalli CN, Kowluru A (2011) Phagocyte-like NADPH oxidase generates ROS in INS 832/13 cells and rat islets: role of protein prenylation. Am J Physiol Regul Integr Comp Physiol 300:756-762

40. Morgan D, Rebelato E, Abdulkader F, Graciano MF, OliveiraEmilo HR et al (2009) Association of NAD(P)H oxidase with glucose-induced insulin secretion by pancreatic beta-cells Endocrinology 150:2197-2201

41. Kowluru A, Kowluru RA (2014) Phagocyte-like NADPH oxidase [Nox2] in cellular dysfunction in models of glucolipotoxicity and diabetes. Biochem Pharmacol 88:275-283

42. Liu Y, Collins C, Kiosses WB, Murray AM, Joshi M et al (2013) A novel pathway spatiotemporally activates Rac1 and redox signaling in response to fluid shear stress. J Cell Biol 201:863-873

43. Servitja JM, Marinissen MJ, Sidhi A, Bustelo XR, Gutkind JS (2003) Rac1 function is required for Src-induced transformation. Evidence of a role for Tiam1 and Vav2 in Rac activation by Src. J Biol Chem 278:34339-34346

44. Garrett TA, Van Buul JD, Burridge K (2007) VEGF-induced Rac1 activation in endothelial cells is regulated by the guanine nucleotide exchange factor Vav2. Exp Cell Res 313:3285-3297

45. Han J, Luby-Phelps K, Das B et al (1998) Role of substrates and products of PI 3-kinase in regulating activation of Rac-related guanosine triphosphatases by Vav. Science 279:558-560

46. McDonald P, Veluthakal R, Kaur H, Kowluru A (2007) Biologically active lipids promote trafficking and membrane association of Rac1 in insulin-secreting INS 832/13 cells. Am J Physiol Cell Physiol 292:C1216-C1220 